ENSAIO

Recebido em: 01/02/2017

Aceito em: 05/07/2017

\title{
Ecumenismo y comunismo. Paralelismos y diferencias de las trayectorias de militancia en los autores de la Revista Paz $e$ Terra (Brasil, 1966-1969)
}

\author{
Ecumenism and Communism. Parallels and Differences of the Trajectories of \\ Militancy in the Authors of the Paz e Terra Magazine (Brazil, 1966-1969)
}

Alejandro PAREDES (aparedes@mendoza-conicet.gob.ar)*

* Professor da Universidad del Aconcagua e Universidad Nacional de Cuyo / Argentina; Pesquisador do CONICET.

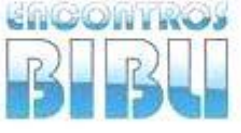

v. 22, n. 50, 2017. p. $223-240$

ISSN 1518-2924

\section{Resumen}

Durante la segunda mitad de la década de 1960, la revista Paz e Terra fue el centro de los debates del movimiento ecuménico brasileño y tuvo fuertes lazos con el Consejo Mundial de Iglesias y el Partido Comunista Brasileño. Este trabajo estudia los temas importantes y las trayectorias políticas y religiosas de los principales autores de la revista. Con respecto a la metodología, se analizaron todos los números de Paz e Terra (1966- 1969) y numerosas fuentes periódicas (entrevistas, obituarios, etcétera) para reconstruir las biografías de los autores. En primer lugar se hizo un análisis de contenido de la revista: se realizó una descripción cualitativa de los números y se usaron nubes de palabras con cada índice. Posteriormente se utilizó el método sociocéntrico del Análisis de Redes Sociales, sumado a un estudio prosopográfico para obtener paralelismos de militancia. Nuestra hipótesis es que en ese contexto (Brasil, 1966-1969), las trayectorias de militancia de comunistas y ecumenistas que publicaron en Paz e Terra presentaron paralelismos (como el humanismo, la persecución política, la formación académica) que les permitió encontrarse en un espacio de debate en común.

Palabras Claves: Militancia político-religiosa. Paz e Terra. Brasil. Década de 1960. Waldo César.

\begin{abstract}
During the second half of the 1960s Paz e Terra magazine was the center of the debates of the Brazilian ecumenical movement and had strong ties with the World Council of Churches and the Brazilian Communist Party. This paper studies the important themes and political and religious trajectories of the main authors of the journal. Regarding the methodology, all the numbers of Paz e Terra (1966-1969) and numerous periodical sources (interviews, obituaries, etc.) were analyzed to reconstruct the biographies of the authors. In the first place a content analysis of the magazine was made: a qualitative description of the numbers was made and clouds of words were used with each index. Subsequently, the sociocentric method of Social Network Analysis was used, together with a prosopographic study to obtain parallels of militancy. Our hypothesis is that in this context (Brazil, 1966-1969), the militancy trajectories of communists and ecumenists who published in Paz e Terra presented parallels (such as humanism, political persecution and academic formation) that allowed them to be in a space for common debate.
\end{abstract}

Keywords: Political and Religious Militancy. Paz e Terra. Brazil. 1960s. Waldo Cesar.

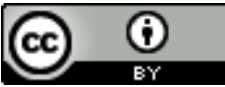

Esta obra está licenciada sob uma Licença Creative Commons. 


\section{INTRODUCCIÓN}

El ecumenismo es un movimiento religioso que busca la unidad de las iglesias cristianas (católicas, protestantes y ortodoxas). La primera organización ecuménica latinoamericana fue la Federación Universal de Estudiantes Cristianos: FUMEC, fundada en 1902, posteriormente nacieron el Comité de Cooperación Latinoamericana: CCLA en 1916, la Unión Latinoamericana de Juventudes Ecuménicas: ULAJE en 1941, la Unidad Evangélica Latinoamericana: UNELAM en 1944, e Iglesia y Sociedad en América Latina (de ahora en más, ISAL) en 1961 (CONCATTI, 2009: 22-23). ISAL aglutinó a los referentes más importantes del ecumenismo latinoamericano (OLIVERA, 2009). Algunos de ellos, como Richard Shaull; Hiber Conteris y Héctor Borrat formaron parte de Paz e Terra. En las décadas de 1960 y 1970 el movimiento ecuménico se identificó con el humanismo crítico, el marxismo y en algunos países, con la Teología de la Liberación (AMAT y LEÓN, 1996: 30). ISAL fue la cuna de la teología de la liberación protestante en tanto que se debatía la pertinencia de una Teología de la Revolución. En este contexto nació Paz e Terra, cuyos autores provienen de distintas iglesias cristianas pero también del Partido Comunista Brasileño. Por esta razón nos preguntamos si esta revista es parte de una red intelectual ecuménica-comunista. Esto sería novedoso para Latinoamérica, porque si bien en Asia existieron militantes religiosos, principalmente del Consejo Mundial de Iglesias, que eran miembros de los partidos comunistas de sus países, en América Latina esto no fue habitual ${ }^{1}$. Para ello indagaremos sobre el origen de la revista, los temas que se debatieron y las trayectorias de militancia de los autores que publicaron más de una vez. Nuestra hipótesis es que en ese contexto (Brasil, 19661969), las trayectorias de militancia de comunistas y ecumenistas que publicaron en Paz e Terra presentaron paralelismos (como el humanismo, la persecución política, la formación académica) que les permitió encontrarse en un espacio de debate en común.

Con respecto a la metodología, la fuente analizada es la totalidad de los números de Paz e Terra (1966- 1969) obtenida en la biblioteca de la Escola Superior de Teologia de São Leopoldo (Brasil) y numerosas fuentes periódicas (entrevistas, obituarios, etcétera) para reconstruir las biografías de los autores. En primer lugar se hizo un análisis de contenido de la revista: se realizó una descripción cualitativa de los números y se usaron nubes de palabras con cada índice. Posteriormente se utilizó la metodología del análisis de redes sociales, en este caso el método sociocéntrico de un grupo de intelectuales sumado a un estudio prosopográfico. El método sociocéntrico consiste en examinar una relación (definida operativamente) en una población conocida en su totalidad. En cuanto al análisis prosopográfico, es un estudio biográfico comparado. Los pasos han sido: 1) obtener la población total (autores de la revista Paz e Terra); 2) definir operativamente la relación (publicaron en el mismo número); 3 ) vincular a los miembros y analizar la red encontrada con UCINET; 4) optar por las trayectorias a investigar (puntos de corte y el editor de la revista) y 5) realizar el análisis prosopográfico y obtener paralelismos de militancia.

\footnotetext{
1 Eduardo Devés (2016) analiza la acción del Consejo Mundial de Iglesias en la llegada de la Teología de la Liberación a Asia; en tanto que Alejandro Paredes (2013) indaga sobre militantes del Consejo Mundial de Iglesias que a su vez eran miembros de Partidos Comunistas asiáticos.
} 


\section{LA REVISTA}

Paz e Terra fue una revista ecuménica amplia o secular que analizaba aspectos de la realidad brasileña e internacional (CESAR, 2007). Apareció durante la última dictadura cívico-militar brasileña. Waldo Cesar, su director describió la situación:

“... já era depois de 1964. Não podíamos escrever, falar por telefone, tudo era vigiado. Nós viajamos, fomos a São Paulo, fomos a Belo Horizonte, fomos a Volta Redonda, lá falei com padres e pessoas que podiam apoiar para organizar o corpo redatorial da revista que era uma coisa expressiva, com gente desde Alceu Amoroso Lima até esses marxistas que eu falei e outros católicos importantes. Então a revista saiu e teve uma repercussão muito grande. Eram 10 mil exemplares a cada 2 meses, esgotava tudo nas bancas. Ela durou de 1966 a 1969" (Editores, 2011).

En el primer número, Paz e Terra se presentó como una revista humanista cristiana, que siguiendo la tradición ecuménica, se muestra abierta al diálogo con otras corrientes de pensamiento.

"PAZ E TERRA é o campo onde os humanismos, as igrejas e os diálogos dos homens de boa vontade superam as diferenças de estrutura e instituição, raça e credo, cultura e partido, para se encontrarem no reconhecimento da necessidade de defender e promover os valores que se ligam à dignidade e a grandeza da vocação do homem”. (PAZ e TERRA N¹, 1966: 4).

Cada número de la revista abordó un tema principal y temas secundarios. Para analizarlas se ha escogido la realización de nubes de palabras, la cual es una representación gráfica de las palabras de un texto, en donde el tamaño de las palabras está vinculado a la frecuencia con la que aparecen. Estos gráficos implican quitar las palabras más utilizadas pero de bajo peso semántico como artículos, preposiciones y conjunciones (HASSAN, HERRERO y GUERRERO, 2010). Se elaboró una nube de palabras con los índices de cada número de la revista con el software online Wordle. 
Gráfico 1: Nubes de palabras de los 10 números de Paz e Terra (1966-1969).
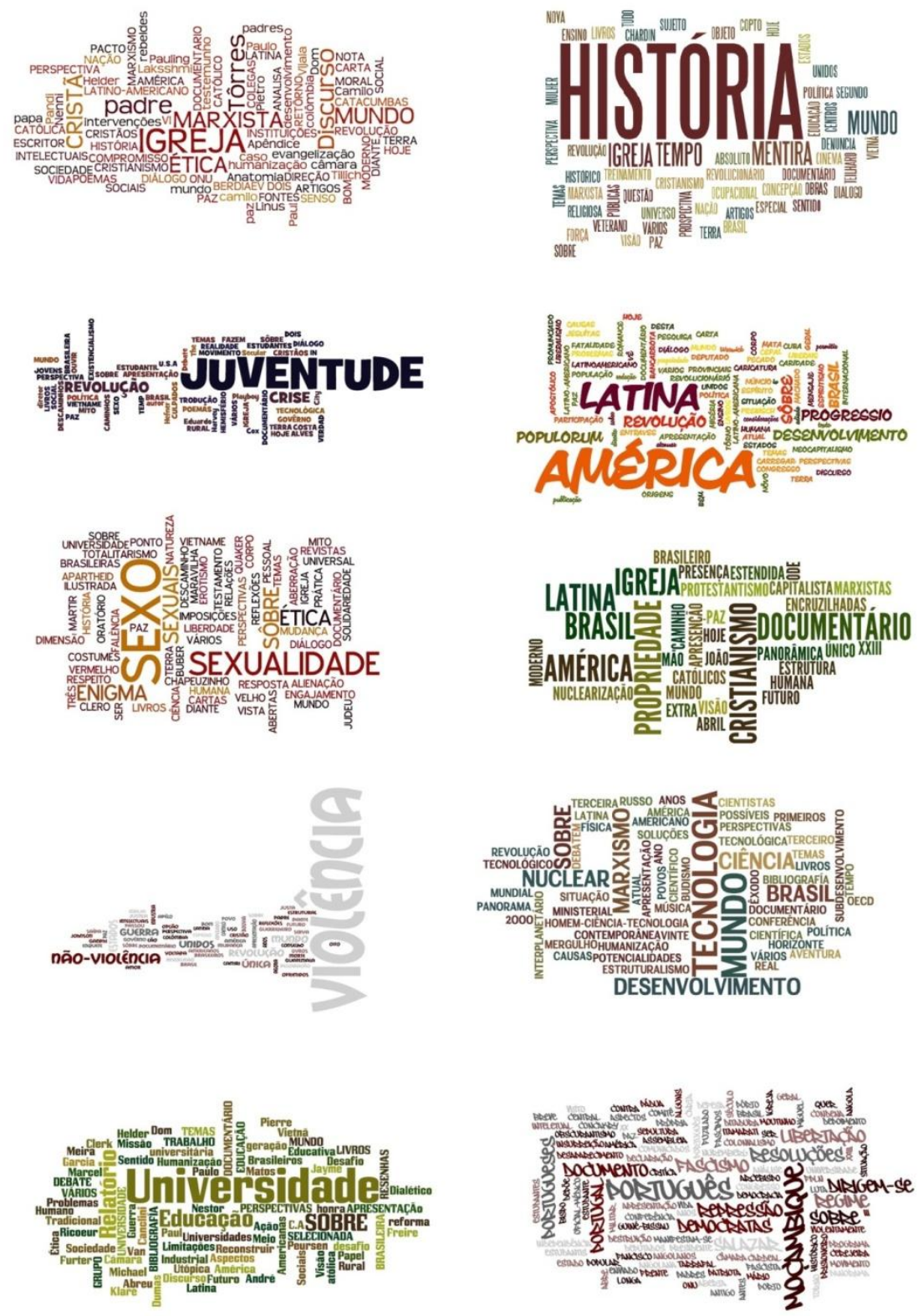

Referencia: Los números están dispuestos del uno al 10 de de izquierda a derecha y de arriba hacia abajo.

Fuente: Revistas Paz e Terra, nubes de palabras con Wordle.

El primer número aparece centrado en los conceptos de Iglesia, ética, marxista y cristiana. Queda claro, además de la perspectiva religiosa, la aceptación del marxismo como punto de partida para la reflexión social. El carácter humanista de Paz e Terra también es postulado en la presentación de la revista, donde afirma: “... nosso ponto de partida é antropológico. Todos os homens. $O$ homen latino- americano com mais ênfase. $O$ brasileiro em especial. Esse homem vive um processo de libertação" ( PAZ e TERRA ํ1, 1966: 4). 
El segundo número se centró en la visión cristiana y marxista de la historia. La palabra Historia es muy predominante sobre el resto. Otras palabras con menor frecuencia pero que se distinguen son Iglesia, Tiempo y Mentira. El tercero estuvo centrado en la juventud. Las palabras que adquirieron mayor significación en la nube de palabras junto a Juventud fueron Crisis y Revolución. Esto se debe a que la revista se preocupará principalmente en la juventud como actor social importante en el cambio social:

"Procuramos, portanto, levar a sério a sadia e desafiante insatisfação da juventude frente a problemas como a guerra, o néo-colonialismo, os regimes de opressão, a fome" (PAZ e TERRA, $\mathrm{N}^{\circ} 3,1967: 3$ ).

En la nube de palabras del cuarto número adquieren relevancia América, Latina, desarrollo, revolución y Populorum Progressio. El número analiza la situación socioeconómica latinoamericana y el rol de los Estados Unidos en ella.

Observando la nube de palabras del quinto número se advierte que la sexualidad fue la temática principal. ¿Cuál es la actitud que debe tomar el progresismo cristiano frente a la sexualidad? A diferencia del conservadurismo que plantea una oposición entre lo sexual y lo religioso, esta corriente se preguntará por el rol alienante de la sexualidad, como dimensión de la libertad humana y los cambios en las prácticas sexuales.

La sexta entrega analiza el rol del cristianismo en América Latina. La revista se opone abiertamente al cristianismo ortodoxo que propone una especie de censura o nueva inquisición.

"Este número de paz e Terra é dedicado ao tema CRISTIANISMO EM QUESTÃO. Com ele não pretendemos oferecer análise definitivo do problema da participação da Igreja na realidade humana e social, mas encaminhar algumas pistas que nos parecem fundamentais" (PAZ e TERRA N $6,1968: 4$ ).

En el número siete la violencia y la vía de la no-violencia para el cambio social adquieren relevancia principal. Como cristianos en el contexto del ascenso revolucionario en América Latina, el posicionamiento ante la violencia era central. La revista presenta autores que defienden la violencia como única salida para terminar la violencia estructural y autores que apoyan movimientos de no violencia al estilo gandhiano. Además incluye una encuesta a cincuenta intelectuales sobre su posición en torno a la adhesión a la violencia.

El octavo volumen enfatiza los conceptos de tecnología, desarrollo, mundo, nuclear y ciencia. Paz e Terra se preocupa "pelas ilimitadas potencialidades da ciência e a nossa incapacidade de vivê-la integralmente e de aplicá-la para o beneficio de toda a humanidade" (PAZ e TERRA N ${ }^{\circ}$, 1968: 4).

En los dos últimos números la persecución contra el director de la revista obstaculizó cada vez más su publicación. En 1967 Waldo Cesar había sido detenido e incomunicado por una semana. Posteriormente en una conferencia que dio sobre su viaje a Colombia a una reunión de ISAL y dónde realizó un artículo sobre Camilo Torres publicado en Paz e Terra ( $\left.\mathrm{N}^{\circ} 1,1966: 243\right)$, fue amenazado por un miembro del público. El 14 de diciembre de 1968, al día siguiente del Ato Institucional no 5, miembros del ejército fueron a buscarlo a su domicilio pero él y su esposa se habían escondido en otra casa por consejos de sus familiares. Se inicia así un periodo en que él intenta continuar con sus actividades pero desde la clandestinidad.

"Eu fiquei três meses fora, vinha ao Rio [de Janeiro] escondido, telefonava com outro nome, era uma coisa horrível. Um ano que você se assusta com a própria sombra, porque pode acontecer, e realmente 
volta e meia apareciam coisas. Era uma perseguição enorme" (Editores, 2011).

Era muy difícil dirigir la revista así y en el número nueve de Paz e Terra, Waldo Cesar publicó su despedida.

"Com este número termino minhas atividades como diretor desta revista. Deixo registrado meu reconhecimento a todos aqueles que confiaram e cooperaram como esta proveitosa experiência cultural e ecumênica - tanto os leitores quanto os membros do conselho de redação - e faço votos de que o debate aberto e honesto entre os homens de boa vontade continue a experiência destes anos" (PAZ e TERRA Nº, 1969: 1).

También Moarcyr Felix, secretario de redacción se solidarizó con Waldo Cesar en su despedida y escribió:

"Circunstâncias de sua vida particular tornam impossível a nosso querido amigo Waldo Cesar continuar dando a esta revista a valiosa colaboração que nos prestou ao longo de quase três anos. Lastimando sua ausência, queremos apresentar-lhe de público os protestos sinceros de nosso reconhecimento, certos de que ele, no curso de sua jornada intelectual, será sempre o mesmo batalhador corajoso pela compreensão entre os homens. De nossa parte, garantimos a continuidade dos propósitos que orientaram o surgimento desta publicação, convencidos que estamos da necessidade cada vez mais premente do encontro e diálogo de todas as tendências do humanismo contemporâneo" (PAZ e TERRA Nº9, 1969: 1).

El número nueve se centró en las palabras: universidad y educación. Fueron seleccionados trabajos que analizan el rol de la universidad en distintos contextos. Entre ellos es interesante el texto de Michael Klare que analiza el rol de las universidades estadounidenses en la guerra de Vietnam.

Lamentablemente las condiciones de trabajo se complicaron aún más. Moacyr Félix también se tuvo que esconder varias veces y estuvo preso en más de una ocasión. Su sufrimiento quedó plasmado en la poesía Canção do Exílio Aqui, de 1977 (UHLY, 2006). El número diez fue un mensaje de esperanza. Asfixiada financieramente por la censura, fue la última edición de Paz e Terra (CUNHA, 2007). El tema central fue el fin de la dictadura de Salazar en Portugal, la más larga hasta el momento del siglo XX. Sus autores eran en su mayoría portugueses aunque, como algunos residían en Brasil, la analogía con la situación brasileña fue constante. Muchos de los artículos eran parte del curso de introducción a la ciencia política promovido por la Pontificia Universidade Católica de São Paulo en septiembre de 1968. La revista analiza también la retracción del colonialismo portugués.

\section{LOS AUTORES}

La revista fue lanzada por Ênio Silveira, miembro del Partido Comunista propietario de la Editora Civilização Brasileira, que era responsable de la edición de la Revista Civilização Brasileira (RCB). Sin embargo el partido comunista brasileño no tenía influencia directa en la revista ya que publicaron con plena libertad una gran cantidad de cristianos de izquierda que no se sentían parte de la revista anterior (PIMENTEL, 2009: 6-7). 
El director responsable era Waldo César y el secretario Moacyr Felix. El consejo de redacción estaba compuesto por Alceu de Amoroso Lima; Frei Pedro Secondi O.P.; Francisco Whitacker Ferreira; Edgar de Godoy Mata Machado; Bernardo Catão; João Dias de Araújo; José Horário Rodrigues; Antônio Otávio Cintra; Jovelino Pereira Ramos; Maria José Brandão Machado; Alfredo Bosi Kühner; Lúcia Ribeiro de Oliveira Ramos; Luiz Alberto Gomes de Souza; Raul Landim Filho; Luiz Eduardo Wanderley; José Paulo Moreira da Fonseca; Glauco Soares de Lima; Padre Francisco Guimarães; Breno Schumann; Jorge César Mota; Esdras Borges Costa y Celso I. de Sylos. Finalmente, los corresponsales extranjeros eran Richard Shaull (Estados Unidos), André Dumas (Francia), Gonzalo Castillo Cárdenas (Colombia), Hiber Conteris y Héctor Borrat de Uruguay.

En la revista se puede observar 112 autores que firmaron 140 artículos.

Tabla 1: Publicaciones por año.

\begin{tabular}{|c|c|c|c|c|c|c|c|c|c|c|l|}
\hline Números de Paz e Terra & 1 & 2 & 3 & 4 & 5 & 6 & 7 & 8 & 9 & 10 & Total \\
\hline Año & 1966 & \multicolumn{3}{|c|}{1967} & \multicolumn{2}{|c|}{1968} & \multicolumn{1}{|c|}{1969} & (1968 \\
\hline $\begin{array}{c}\mathbf{N}^{\circ} \text { publicaciones por } \\
\text { número de Paz e Terra }\end{array}$ & 24 & 14 & 11 & 16 & 16 & 8 & 18 & 12 & 11 & 10 & 140 \\
\hline
\end{tabular}

Fuente: Dados de la investigación.

Con respecto a la renovación de los autores, el siguiente gráfico analiza la vinculación entre las entregas de Paz e Terra a partir de la publicación de autores en común.

Gráfico 2: Vinculación de los números a través de sus autores.

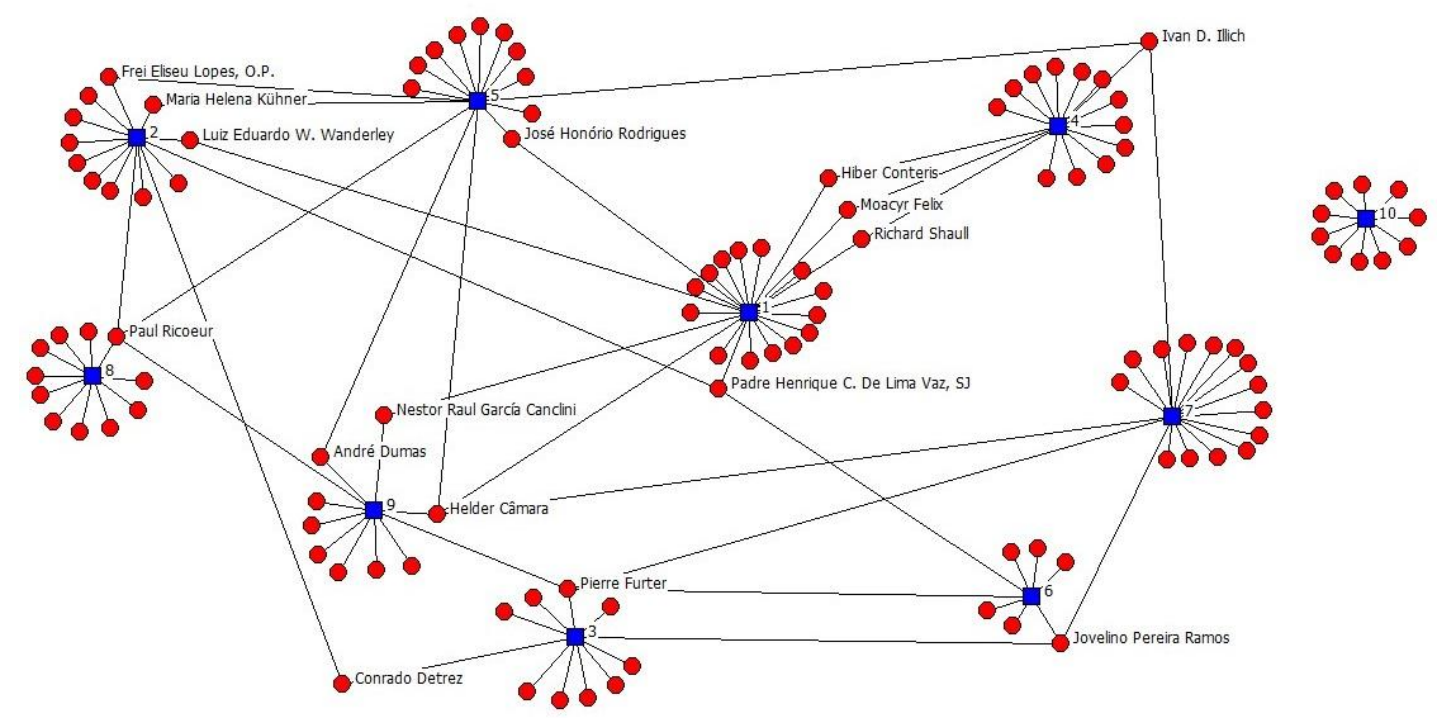

Nota: Colores de los nodos: azul (volumen de La revista), rojo (autores).

Fuente: revistas Paz e Terra, software UCINET.

Solamente en el último número de la revista no intervinieron autores que habían escrito en otras ediciones. De todos modos, los autores que han escrito en más de un ejemplar de la revista son pocos (en el gráfico son los nodos que tienen nombre). La tabla que sigue a continuación detalla la cantidad de veces que publicó cada autor. 
Tabla 2: Autores y la frecuencia de publicación.

\begin{tabular}{|c|c|c|c|}
\hline \multirow{2}{*}{$\begin{array}{c}N^{\circ} \text { de } \\
\text { artículos }\end{array}$} & \multirow{2}{*}{ Autor } & \multicolumn{2}{|c|}{ Total } \\
\hline & & $\mathbf{N}$ & $\%$ \\
\hline 4 & Helder Câmara, Pierre Furter, Paul Ricoeur & 3 & 2,5 \\
\hline 3 & $\begin{array}{l}\text { Ivan D. Illich, Jovelino Pereira Ramos, Moacyr Felix, Henrique C. De Lima } \\
\text { Vaz }\end{array}$ & 4 & 3,5 \\
\hline 2 & $\begin{array}{l}\text { André Dumas, Hiber Conteris, J. Leite Lopes, José Honório Rodrigues, Luiz } \\
\text { Alberto Gómes De Sousa, Conrado Detrez, Frei Eliseu Lopes O.P., Maria } \\
\text { Helena Kühner, Nestor Raul García Canclini, Luiz Eduardo Wanderley, } \\
\text { Richard Shaull }\end{array}$ & 11 & 10 \\
\hline 1 & $\begin{array}{l}\text { A. Bernardo Peres, Afonso Henriques Sacramento, Alceu de Amoroso Lima, } \\
\text { Alfredo Marques, Anders Johansson, Ângela Neves, Augusto Aragão, Basil } \\
\text { Davidson, Bertrand Russell, Brady Tyson, C.A. Van Peursen, Carl Oglesby, } \\
\text { Carlos Drummond De Andrade, Celso Furtado, Cláudio Santoro, Claudius, } \\
\text { Conrado Eggers Lan, D. Antônia Ferreira Gomes, Danilo Zolo, Dep Edgar } \\
\text { Godoy Mata Machado, Eduardo Alves Da Costa, Ernst Bloch, Fernando } \\
\text { Mendes Vianna, Francisco C. Rolim, Francisco Carvalho, Frei Carlos Alberto } \\
\text { Cristo, Frei Francisco De Aráujo O.P., Frei Pedro Secondi Op, Frei Romeu } \\
\text { Dale O. P., George Hourdin, Gonzalo Castilho C., H. M. Nussenzveig, Harvey } \\
\text { Cox, Hector Borrat, Hugh Hefne, J. Kosinski De Cavalcanti, Jacqueline Skiles, } \\
\text { Jayme Abreu, Jean Brun, Jean Lacorix, Joaquim Barradas De Carvalho, John } \\
\text { Gerassi, John M. Swomley Jr, Jose Itamar De Freitas, Karl Barth, León } \\
\text { Poliakov, Leônidas R. Xausa, Linus Pauling, Luiz Maranhão, Marcel De Clerk, } \\
\text { Maria Antônia Fiadeira, Mário Carvalho De Jesus, Mário Moutinho De Pádua, } \\
\text { Martin Luther King, Meira Matos, Michael Klare, Michel Dufrenne, Michel } \\
\text { Verret, Miguel Urbano Rodrigues, Monsenhor Germán Guzman, Murillo } \\
\text { Nunes De Azevedo, Nelson Pilosof, Noronha Filho, Oscar Maggiolo, } \\
\text { Raymundo Ozanam De Andrade Sj, Pablo Piacentini, Padre Camilo Torres, } \\
\text { Padre Thomas Melville, Pamela Mills, Paul Lehmann, Paul Louis Landsberg, } \\
\text { Paul Tilich, Paulo Freire, Paulo VI, Pietro Nenni, Mario Schenberg, R. Simon } \\
\text { S.D.B., Ramón Ramirez Gomez, Raymond Domergue OFM, Régis Debray, } \\
\text { Vijaia Laksshmi Pandit, Vitor Ramos, Waldo Cesar, Warwick Esteban, Kerr, } \\
\text { Yvan Simonis, Yvon Brés, Leandro Konder, Lauro de Oliveira Lima, Julio de } \\
\text { Santa Ana, Otto Maria Carpeaux, Alberto Salvá Contel, Jack Raymond, Donald }\end{array}$ & 94 & 84 \\
\hline & Total & 112 & 100 \\
\hline
\end{tabular}

Nota: en negrita los autores que eran parte del equipo de redacción

Fuente: Dados de la investigación.

Comparando a los autores con los miembros del equipo de redacción puede observarse una muy baja endogamia. La gran mayoría (el 86,54\%) publicaron una sola vez. Waldo César, director de la revista, sólo firmó una publicación, en tanto que Moacyr Felix, quien fue secretario del director y luego director, publicó tres artículos.

\section{LAS TRAYECTORIAS DE MILITANCIA}

Analizaremos las biografías de los autores que publicaron más de una vez en la revista y a Waldo César que era el director. A pie de página se ha listado las publicaciones más importante de cada autor para ilustrar sus preocupaciones teóricas.

Los que publicaron más veces, fueron Pierre Furter, Helder Câmara y Paul Ricoeur. 
Pierre Furter: nació en 1931 en Suiza. Estudió Filosofía y pedagogía en la Universidad de Lausanne, en Nauchâtel ${ }^{2}$. Se especializó en literatura comparada en Lisboa, Zurich y Recife. Después de doctorarse en filosofía de la educación, trabajó durante largo tiempo en América Latina, primero en Brasil, luego en Venezuela. es profesor en la Universidad de Ginebra, presidente de la Sociedad Suiza para la Investigación Educativa y vicepresidente de la Asociación Francófona de Educación Comparada. Ha sido experto de la UNESCO, consultor del Instituto Internacional de Planificación Educativa de París y representante de la Confederación Suiza en diversas conferencias internacionales sobre educación.

Helder Câmara (1909-1999) uno de los más destacados teólogos de la liberación. En 1931 fue ordenado sacerdote, en 1936 fue trasladado a Río de Janeiro, donde se preocupó por las condiciones de vida de los habitantes de las favelas. En 1952 fue nombrado obispo auxiliar de Río de Janeiro. Impulsó la creación de la Conferencia Nacional de Obispos Brasileños y posteriormente la Conferencia Episcopal Latinoamericana (CELAM). En 1962, participó del Concilio Vaticano II. En 1964 fue arzobispo de Olinda y Recife. A partir de allí su visibilidad en los medios de comunicación donde defendía la llamada Opción por los pobres, le costó la persecución del régimen militar. En 1968 y 1969, años en que publicó en Paz e Terra, su casa fue ametrallada y luego fue asesinado uno de sus colaboradores más cercano, el sacerdote Antonio Henrique Pereira Neto. En 1970 el régimen lo acusó de ser parte del comunismo internacional y le prohibió aparecer en medios de comunicación o que sea nombrado por ellos. Se jubiló en 1984 con 75 años 3 .

Paul Ricoeur (1913-2005) filósofo francés y pensador protestante. Estuvo preso por cuatro años durante la segunda guerra mundial. Fue profesor en la Sorbonne, en Estrasburgo y decano de la Facultad de Letras de la Universidad de Nanterre (LAFUENTE, 1998). Colaboró en la revista existencialista cristiana Esprit junto a André Dumas. Se abocó a analizar el rol social del cristianismo ${ }^{4}$.

Le siguen en cantidad de publicaciones Ivan Illich, Jovelino Pereira Ramos Moacyr Felix y Henrique De Lima Vaz.

Ivan D. Illich (1926-2002) Pedagogo, nació en Austria. Vivió en los Estados Unidos, en 1956 se trasladó a Puerto Rico para ser vicerrector de la Universidad de Ponce (católica). Junto a Paulo Freire, asesoró la política educativa del gobierno boliviano de Alfredo Ovando Candía. En los sesenta fundó en Cuernava, México y

\footnotetext{
2 Autor de: Juventude e tempo presente (1967), Educación y vida (1996)

${ }^{3}$ Muchos de sus sermones fueron publicados en Revolução dentro da paz (1968). Otras publicaciones son: El Evangelio con Dom Helder, El desierto es fértil, Mil razones para vivir, Las conversiones de un obispo, Cristianismo, socialismo, capitalismo, ¿Quién soy yo?, Espiral de violencia, La rebelión de los economistas, Revolución de los no violentos: una iniciativa, La Iglesia y el desarrollo de América Latina, Para llegar a tiempo y Pobreza, abundancia y solidaridad.

4 Autor de: Karl Jaspers et la philosophie de l'existence (con Mikel Dufrenne, 1947); Gabriel Marcel et Karl Jaspers (1948); Philosophie de la volonté (dos tomos, 1950 y 1960); Histoire et vérité (1955); De l'interprétation. Essai sur Sigmund Freud (1965); Entretiens avec Gabriel Marcel (1968); Essais d'herméneutique (dos tomos, 1969 y 1986); La métaphore vive (1975); Les cultures et le temps (1975); Temps et récit (tres tomos, 1983, 1984 y 1985); À l'école de la phénoménologie (1986); Le mal. Un défi à la philosophie et à la théologie (1986); Soi-même comme un autre (1990); Réflexion faite. Autobiographie intellectuelle (1995); Le juste (dos tomos, 1995 y 2001); L'idéologie et l'utopie (1997); Amour et justice (1997); Ce qui nous fait penser (con Jean-Pierre Changeux, 1998) Penser la Bible (con André LaCocque, 1998); Lectures (tres tomos, 1999); La mémoire, l'histoire, l'oubli (2000); L'herméneutique biblique (2000); La lutte pour la reconnaissance et l'économie du don (2002); Parcours de la reconnaissance. Trois études (2004); Sur la traduction (2004) y Écrits et conférences (dos tomos, 2008 y 2010)
} 
Petrópolis, Brasil centros de formación intercultural destinado a habilitar europeos y norteamericanos que trabajarían en América Latina. Era considerado entre los intelectuales anglosajones como uno de los mayores especialistas en Latinoamérica ${ }^{5}$.

Jovelino Pereira Ramos, Estudió Teología en el Seminario Prebisteriano de Campinas, y posteriormente en la Universidad de Yale (en Estados Unidos) entre 1960 y 1962. Después del golpe militar brasileño debió exiliarse. Tradujo "Cristo y Cultura" de Richard Niebuhr, que fue publicado por la editora Paz e Terra. Publicó en la revista desde los Estados Unidos, donde vivía.

Moacyr Felix (1926-2005). Poeta, se licenció en derecho (Universidad Católica de Rio de Janeiro). En la década de 1950, fue colaborador del diario del Partido Comunista Brasileiro "Para todos", dirigido por Jorge Amado y Oscar Niemeyer (S/A, 26/10/2005). Entre 1950 e 1953 estudió Letras en París. Entre 1963 y 1971, dirigió la Coleção Poesia Hoje, da Civilização Brasileira. También en 1963 fue uno de los fundadores del Comando de Trabalhadores Intelectuais (CTI) donde se desempeñó como Secretario General. El CTI fue una agremiación cultural cuya diversidad manifestaba las tensiones políticas de la sociedad brasilera del pre-golpe de 1964 (CZAJKA, 2011: 63-64). Fue uno de los ideólogos de la revista Paz e Terra, de la cual fue director en sus últimos números6.

Henrique C. De Lima Vaz (1921-2002) Nació en Minas Gerais. Con solo 16 años ingresó al seminario y se ordenó Padre Jesuita. Licenciado en Filosofía (Colegio de Anchieta, Nova Friburgo). Doctor en Teología (Universidad Gregoriana de Roma). Profesor de Filosofía en la Facultad de Nossa Senhora Medianeira (Nova Friburgo) y en la Universidade Federal de Minas Gerais (Belo Horizonte). Fue el mentor del movimiento Juventud Universitaria Católica (JUC). Autor de varios trabajos filosóficos ${ }^{7}$ (Mac Dowell, 2002).

Con dos publicaciones se encuentran: André Dumas, Hiber Conteris, Leite Lopes, José Honório Rodrigues, Luiz Alberto Gómes De Sousa, Conrado Detrez, Eliseu Lopes O.P., Maria Helena Kühner, Nestor García Canclini, Luiz Eduardo Wanderley, Richard Shaull.

André Dumas (1918-1996) Pastor protestante. Estudió teología durante la segunda guerra mundial y era miembro de la organización protestante CIMADE (Comite inter-mouvements aupres des evacues) que socorrió a judíos internados en el campo de concentración de Rivesaltes, cerca de la frontera española. Otorgó documentos falsos a varios de ellos para que pudieran escapar y por eso en 1994 recibió el título de Justo

\footnotetext{
${ }^{5}$ Autor de: La escuela, esa vieja y gorda vaca sagrada (1968), Una sociedad sin escuela (1971), Herramientas para la convivencialidad (1973), Energía y equidad (1973), Némesis médica: la expropiación de la salud (1975), Educación sin escuelas (1975), La sociedad desescolarizada (1978), La escuela y la represión de nuestros hijos (1979) -escrita en colaboración con Hildegard Lüning-, Shadow-work (1981), Producir (1982), Ecofilosofías (1984), En América Latina, ¿para qué sirve la escuela? (1985), La educación (1986) -con Paulo Freire-, y H2O y las aguas del olvido (1989).

${ }^{6}$ Autor de: Cubo de Treva (1948); Lenda e Areia (1950); Itinerário de uma Tarde (1953); O pão e o Vinho (1959); Canto para as Transformações do Homem (1964); Um Poeta na Cidade e no Tempo (1966); Canção do Exílio Aqui (1977); Neste Lençol (1977); Invenção de Crença e Descrença (1978); Em Nome da Vida (1981); Encontros com a Civilização Brasileira (1981); Singular Plural (1988); Introdução a Escombros (1998, que obtuvo varios premios).

${ }^{7}$ Autor de: Universo científico e visão cristã em Teilhard de Chardin (1967); Ontologia e história (1968); Escritos de filosofia (siete tomos publicados entre 1986 y 2002); Antropologia filosófica (tres tomos publicados entre 1991 y 1997); Experiência mística e filosofia na tradição ocidental (2000); Ética e direito (2002); Contemplação e dialética nos diálogos platônicos (2012) y A formação do pensamento de Hegel (2014).
} 
entre las Naciones (RIGHTEOUS AMONG THE NATIONS DEPARTMENT, 1994). Entre 1961 y 1984 fue Profesor en la facultad de Teología Protestante de París y en la Facultad de teología de Estrasburgo. Entre 1973 y 1975 fue decano de la Facultad Protestante de Teología de París (PAZ e TERRA n 5) ${ }^{8}$.

Hiber Conteris escritor uruguayo nacido en 1933. Estudió teología, filosofía y letras. Trabajó como obrero en Brasil en una experiencia de relacionamiento y testimonio cristiano con el mundo obrero. Fue profesor en Bolivia, Argentina y Uruguay. Fue secretario de estudios de la junta latinoamericana de ISAL. Escribió obras de teatro, cuento y novelas. Colaboró con las revistas uruguayas Marcha y Cristianismos y Sociedad. La dictadura cívico-militar uruguaya lo encarceló nueve años y luego tuvo que exiliarse los Estados Unidos donde fue profesor en la Universidad de Madison, Alfred y Arizona $^{9}$ (CERVANTES-ORTIZ, 2014).

José Leite Lopes (1918-2006) ingeniero químico, licenciado en física (Universidade federal do Rio de Janeiro), doctor en física (Princenton University, EEUU) $\mathrm{y}$ doctor en ciencias (Universidade federal do Rio de Janeiro) donde es profesor ${ }^{10}$. Acusado de participar en una conspiración comunista, fue perseguido por el gobierno militar y debió exiliarse en Francia. Retornó a Brasil en 1968 y fue Director del Instituto de Física de la Universidade Federal do Rio de Janeiro, pero en 1969 fue jubilado compulsivamente. Volvió a Europa y fue profesor en la Universidade de Strasbourg (1970-1985). En 1985 regresó a Brasil para trabajar en el Centro Brasileiro de Pesquisas Físicas, centro que él mismo había contribuido a fundar y que dirigió. Recibió la Medalla Felipe Carneiro de la Comisión Nacional de Energía Nuclear de Brasil (1988); la Ordre des Palmes Académiques (1989) y la Ordre National du Mérite (1989) de Francia; Premio Álvaro Alberto Nacional de Ciencias (1989), el Premio de Ciencia y Tecnología de México (1993) y el Premio de Ciencias de la UNESCO (1999) (CARUSO, 2009).

José Honório Rodrigues (1913-1987). Gran historiador brasileño. Fue director de la Divisão de Obras Raras e Publicações da Biblioteca Nacional (1946-1958), director de la Seção de Pesquisas do Instituto Rio Branco, do Ministério das Relações Exteriores (1948-1951); del Archivo Nacional de Brasil (1958-1964); del Instituto Brasileiro de Relações Internacionais (1964-1968) y de la Revista Brasileira de Política Internacional (misma fecha). Recibió el Premio de Erudição da Academia Brasileira de Letras (1937), el Prêmio Clio de Historiografia da Academia Paulista de Letras (1980), el Premio de História do Instituto Nacional do Livro (1980) y la Medalha do Congresso Nacional (1980). Fue profesor de distintos aspectos relacionado con la historia en el Instituto Rio Branco, do Ministério das Relações Exteriores; la Pontifícia Universidade Católica do Rio

\footnotetext{
8 Autor de: Ma sœur Anne (1936); Théologie du corps (1965-1966); Le contrôle des Naissances (1965); Une Théologie de la réalité: Dietrich Bonhoeffer (1968); Ces mots qui nous font croire et douter (1971); Prospective et prophétie: Les Églises dans la société industrielle (1972); Théologies politiques et vie de l'Église (1977); Nommer Dieu (1980); Marie de Nazareth (con Francine Dumas, 1989); Cent prières possibles (1991). ${ }_{9}^{9}$ Autor de: El diez por ciento de vida (1986), La Diana en el crepúsculo (1986), Información sobre la Ruta 1 (1987), El intruso (1987), Holiday Inn (1988), El breve verano de Nefertiti (1996), ¿Qué desea cenar? (1996), El cielo puede esperar (1998), Round Trip. Viaje regresivo (1998), Mi largo adiós a Raymond Chandler (1999), Rastros de ceniza (2001), Oscura memoria del sur (2002) y Onetti en el espejo (2005).

${ }^{10}$ Autor de: Ciência e libertação (1969); Einstein e outros ensaios (1958); Ciência e desenvolvimento (1987); Ciência e liberdade. Escritos sobre ciência e educação no Brasil (1998).
} 
de Janeiro; en la Universidade Federal Fluminense; en la Universidade Federal do Rio de Janeiro y varias universidades estadounidenses ${ }^{11}$.

Luiz Alberto Gómes De Sousa Licenciado en Derecho (Pontificia Universidade Católica do Rio Grande do sul) concluyó la maestría en Ciencia Política de la Facultad Latinoamericana de Ciencias Sociales y doctorado en Sociología por la Universidad de París. En 1950 formaba parte de la JEC (Juventude Estudantil Católica) y en 1954 pasó a la JUC (Uuventude Universitária Católica) de Porto Alegre. Desde 1556 a 1958 integró la JUC nacional. En 1958 participó en un encuentro internacional en Dakar donde conoció a miembros de la JUC argentina y paraguaya. Fue Secretario General de la JEC internacional de 1959 a 1961, en la sede en París. De regreso en Brasil, siguió en contacto con la JUC hasta el golpe de 1964, cuando fue expulsado (TOURIS, 2010). Es funcionario de la Comisión Económica para América Latina y el Caribe (CEPAL), jefe de la Oficina para América Latina y el Caribe Departamento de Desarrollo de la FAO en Roma. Fue director del Centro de Estadística Religiosa e Investigaciones Sociales y actualmente es director del Programa de Estudios Avanzados en Ciencias y Religión de la Universidade Cândido Mendes ${ }^{12}$.

Conrado Detrez (1937-1985) belga, diplomado en Teología (Universidade de Louvain) y en Lengua y Literatura francesa (Universidad de Nancy). En 1962 se mudó a Brasil donde fue profesor y periodista. Detrez llega a Portugal en 1975, aunque en ruptura con el pensamiento de izquierda, como lo deja en claro en Les noms de la tribu (1981). Se enfrentó al gobierno militar participando en el "Movimento Democrático Brasileiro" y en 1967 fue preso y torturado por seis días. De todos modos nunca adhirió a la lucha armada (DOMINGUES DE ALMEIDA, 2015). Se mudó a Francia pero regresó por lo que fue encarcelado dos años. En 1980 fue indultado por el gobierno brasileño y regresó pero en 1982, en su retorno al socialismo pidió la ciudadanía francesa, y fue nombrado agregado cultural y científico de la Embajada de Francia en Nicaragua sacudida por luchas sociales y políticas. En 1985 murió de Sida13.

Frei Eliseu Lopes O.P., Brasileño. Ingresó temprano a la congragación de los Lazaristas. Fue profesor en los seminarios mayores de Fortaleza, Sao Luís y Salvador. Pasó a pertenecer a la orden de los pregadores (Dominicanos) en 1953 y allí fue director de la Escola Apostólica (Juiz de Fora) y Prior del Convento de Rio de Janeiro. En 1968, asistió al "Encontro da Juventude Operária Católica (JOC)", en Recife (DA MATA, 2007). Fue miembro del movimiento ecuménico (CORREIO DA MANHÃ, 21/07/1966). Adquirió relevancia en el mundo católico por sus artículos, conferencias y oraciones.

Maria Helena Kühner, (1933-) Licenciada en Filosofía y letras (Pontifícia Universidade Católica do Rio de Janeiro). Montó con su marido un colegio primario y

\footnotetext{
${ }^{11}$ Autor de Brasil e África: outro horizonte (1961); Aspirações nacionais (1963); História e historiadores do Brasil (1965), Conciliação e Reforma no Brasil (1965); Teoria da História do Brasil (1949). Civilização holandesa no Brasil (1940); Historiografia e bibliografia do domínio holandês no Brasil (1949); As fontes da História do Brasil na Europa (1950); A pesquisa histórica no Brasil (1952); Brasil. Período colonial (1953); O continente do Rio Grande (1954); Interesse nacional e política externa (1966); Vida e história ( 1966); História e historiografia ( 1970); A Assembléia Constituinte de 1823 (1974); Independência: revolução e contra-revolução ( 5 vols. ( 1976); História corpo do tempo (1976); O Conselho de Estado. O Quinto Poder? (1978).

12 Autor de Classes Populares e Igreja nos Caminhos da História (1982); A Juc: Os Estudantes Católicos e a Política (1984); A Utopia Surgindo no Meio de Nós (2003); Do Vaticano II a um Novo Concílio? o Olhar de um Cristão Leigo Sobre a Igreja (2004).

13 Autor de Révolution dans la paix (1970); Pour la libération du Brésil (1970); Les Mouvements révolutionnaitres en Amérique latine (1972); Ludo (1974); Les Plumes du coq (1975); L'Herbe à brûler (1978); La Lutte finale (1980); Le Dragueur de Dieu (1980); Les Noms de la tribu (1981); La Guerre blanche (1982); Le Mâle apôtre (1982); La Ceinture de feu (1984); La Mélancolie du voyeur (1986)
} 
secundario del que fue Directora. Escribió ensayos, artículos, obras de teatro, reseñas y traducciones (1962-1970). Durante la dictadura cívico -militar brasileña se le prohibió ser contratada por el Estado debido a su vinculación con el movimiento estudiantil y obrero (KÜHNER, 2003). Entre 1991 y 1994 fue Directora de la Revista de Teatro SBAT (Sociedade Brasileira de Autores Teatrais) ${ }^{14}$.

Nestor Raul García Canclini (1939-). Argentino, doctor en Letras por la Universidad Nacional de La Plata y por la Universidad de París. Fue profesor en la Universidad de La Plata, en la Universidad de Buenos Aires y en la Universidad Autónoma Metropolitana. Fue miembro activo del movimiento de estudiantes cristianos de Argentina (MEC) y de ISAL. Ha sido profesor visitante de diversas universidades, entre ellas las de Nápoles, Austin, Stanford, Barcelona y São Paulo ${ }^{15}$ (INFOAMÉRICA, $\mathrm{S} / \mathrm{F})$.

Luiz Eduardo Wanderley, Licenciado en Ciencias Jurídicas y Sociales (Universidade de São Paulo), miembro del equipo nacional de Juventud Universitaria Católica de Brasil (1960-1962) y del secretariado nacional de la Acción Católica de Brasil (1962-1963). Doctor en Ciencias Sociales (Universidad de São Paulo) y Postdoctor por la École des Hautes Études en Sciences Sociales, Paris. Fue profesor en la Pontifícia Universidade Católica de São Paulo y en la Universidad de São Paulo. Fue Director del Instituto de Relações Latino-Americanas en la Pontificia Universidad Católica de São Paulo (1981-1984) y Rector en esa misma universidad (1984-1988). Miembro del Directorio del Centro Ecumênico de Educação e Evangelização Populares de São Paulo desde 1989 y de la ONG Ação Educativa no período de 1999-200016.

Richard Shaull (1919-2002) Estadounidense, licenciado y doctor en teología, profesor del Seminario Prebisteriano de Princeton (Estados Unidos) y de Campinas (Brasil). En 1942 viajó a Colombia como Pastor misionero, en 1952 se mudó a Brasil. Si bien en un primer momento buscó combatir al comunismo a través de la iglesia evangélica, su acercamiento a la realidad latinoamericana lo hizo concentrarse cada vez en los problemas sociales y la justicia social ${ }^{17}$. Fruto de ello en 1955 publicó Encounter

\footnotetext{
14 Autora de: Teatro em tempo de sintese (1971); Opções da esquerda (1972); Teatro Popular - Uma experiência (1974); O Desafio atual da mulher (1977); O Teatro de Revista e a Questão da Cultura Nacional e Popular (1979); O-que-se-mostra e o-que-se-esconde (1986); A Comunicação Teatral: 1980-83 (1984); Será uma vez (1986); A Linguagem do teatro hoje (1987); É (1988); Teatro - Espelho e Resposta (Con Gilberto Kühner 1989); A Transgressão do Feminino (1989); Homem / Mulher - Uma relação em mudança (vários autores 1994); Geschlechter - Performance, Pathos, Politik (vários autores 1997); O Fio de Ariadne (1998); Opinião (2001). Obras de teatro: Foto de Crepúsculo (1971); Anchieta (1972); A Menina que buscava o Sol (1975); Represa (1980); Com o Suor do Nosso Rosto (1985)

${ }^{15}$ Autor de: Arte popular y sociedad en América Latina (1977); La producción simbólica. Teoría y método en sociología del arte (1979); Las culturas populares en el capitalismo (1982); ¿De qué estamos hablando cuando hablamos de lo popular? (1986); Cultura transnacional y culturas populares (con R. Roncagliolo, 1988); Culturas híbridas. Estrategias para entrar y salir de la modernidad (1990); Cultura y Comunicación: entre lo global y lo local (1997); Las industrias culturales en la integración latinoaméricana (con Carlos Moneta, 1999); La globalización imaginada (1999); Imaginarios Urbanos (1999); Consumidores e cidadãos. Conflitos multiculturais da globalizacao (1999) y Latinoamericanos buscando lugar en este siglo (2002).

16 Autor de: Educar para Transformar - educação popular, Igreja Católica e política no Movimento de Educação de Base (1984); O que universidade (1983); Educação popular: metamorfoses e veredas (2010)

${ }^{17}$ Autor de: O christianismo e a revolução social (1953); Encounter with revolution (1955); Containment and change (junto a Carl Oglesby 1967); Liberation and change (con G. Gutierrez, 1977); Heralds of a new reformation: the poor of South and North America (1984); De dentro do furacão: Richard Shaull e os primórdios da Teologia da Libertação (1985); A reforma protestante e a teologia da libertação: perspectivas para os desafios da atualidade (1993); Pentecostalismo e futuro das igrejas cristãs: promessas e desafios (con W. Cesar, 1999); Pentecostalism and the Future of the Christian Churches: Promises, Limitations, Challenges (con W. Cesar, 2000); Surpreendido pela graça (2003).
} 
With Revolution. En 1955 el Consejo Mundial de Iglesias lo invitó a las conferencias Christian Responsibility Toward Areas Of Rapid Social Change y él fue con un grupo de estudiantes de movimientos cristianos que luego serán la base de ISAL: José Miguez Bonino, Julio de Santa Ana, Gonzalo Castillo Cárdenas, Rubem Alves, Waldo Cesar, Dick Schatier, Jether Ramalho e Hiber Conteris. Directamente implicado en el nacimiento de ISAL y de la teología de la liberación, hacia 1967 comenzó a discutir en el Consejo Mundial de Iglesias y en el Seminario Prebisteriano de Princeton, si los cristianos debían unirse a la guerra de guerrillas. Hacia el año siguiente junto a otros miembros de ISAL propusieron el término de "Liberación" en lugar de "revolución" para hablar de la situación latinoamericana (SMITH, 1991: 115-117). Perseguido por la dictadura brasileña, tuvo que exiliarse (SOTO, 2011: 65).

Finalmente entre los autores con una publicación sólo nos detendremos en el director de la revista.

Waldo Aranha Lenz Cesar (1922-2007), graduado en el Instituto Ecuménico del Consejo Mundial de Iglesias (Ginebra). Fue secretario ejecutivo del Sector de Responsabilidad Social de la Iglesia de la Confederación Evangélica de Brasil entre 1955 y 1964, pero fue alejado por las repercusiones de la Conferencia del Nordeste en 1962 con el tema Cristo e o Processo Revolucionário Brasileiro (PIMENTEL, 2009). Fue uno de los fundadores del Centro Ecuménico de Información, Secretario adjunto de ISAL, Director de la revista Paz e Terra (1966-1969), miembro del equipo de redacción de Cristianismo y Sociedad (Uruguay) y coordinador de la Campaña Mundial contra el hambre de FAO (institución a la que también estuvo vinculado Luiz Alberto Gómes De Sousa) entre 1979 y 198718 (EDITORES, 2011).

\section{CONCLUSIONES}

Paz e Terra nació de la vinculación entre el movimiento ecuménico y el Partido Comunista Brasileño. Los que dirigieron la revista son representantes de ese matrimonio intelectual: Waldo César era un líder del movimiento ecuménico y Moacyr Felix había sido colaborador en el diario del Partido Comunista Brasileiro y uno de los fundadores del Comando de Trabalhadores Intelectuais.

De todos modo la baja endogamia de la revista (el 84\% publicó solo una vez) manifiesta que la temática fue un criterio predominante sobre los potenciales autores y permite sospechar que no nos encontramos ante un núcleo de una red del que $\operatorname{Paz} e$ Terra era portavoz sino más bien un espacio convocante a partir del cual se buscaban autores entre las dos corrientes intelectuales (ecumenismo y comunismo) según el tópico de cada entrega.

Si tomamos a los autores que publicaron más de una vez, se observan trayectorias de militancia distintas algunas en el campo religioso (ecuménico o eclesial) otras en el partido comunista (muchos de los intelectuales tienen paralelamente esta militancia). Sin embargo, al analizar estos dos grupos como un todo, es notorio los puntos en común de las trayectorias, todos eran intelectuales muy formados, no todos cristianos. Los no-cristianos eran marxistas. En su gran mayoría fueron perseguidos por la dictadura cívico-militar brasileña. El grupo está conformado por religiosos (Câmara; Lima Vaz; Dumas; Eliseu Lopes; Shaull y Pereira Ramos); escritores (Felix; Conteris; Detrez y Kühner); cientistas sociales (García Canclini; Wanderley; Illich y Waldo Cesar);

18 Autor de: Para uma sociologia do protestantismo brasileiro (1973), Pentecostalismo e futuro das igrejas cristãs (con Richard Shaull 1999), Tenente Pacífico (202) 
filósofos (Ricoeur y Furter); un abogado (Gómes De Sousa); un historiador (Honório Rodrigues) y un físico (Leite Lopes).

En el grupo analizado solo hay una mujer, Maria Helena Kühner. En total son once brasileños y ocho extranjeros, de los cuales cuatro vivieron en Brasil (Detrez, Shaull, Illich y Conteris), dos estaban vinculados a la revista francesa cristiana humanista Sprit (Dumas y Ricoeur) y los últimos dos al movimiento ecuménico (Illich y García Canclini). Es un grupo claramente intelectual en el que todos realizaron carreras universitarias y trece de ellos, algún curso de posgrado.

En cuanto a las trayectorias religiosas, solo seis de ellos no tuvieron cargos en ningún movimiento religioso. En algunos casos, como en el de Moacyr Felix, se debió a su ateísmo y adhesión al partido comunista brasileño. Ocho tuvieron cargos del movimiento ecuménico, siete eran parte de la jerarquía religiosa de sus iglesias (pastores, sacerdotes u obispos).

Con respecto a las trayectorias políticas, diez sufrieron algún tipo de persecución, seis tuvieron que exiliarse (en algunos casos exilio interno) y tres estuvieron presos en Brasil, Conteris en Uruguay y Ricoeur en Europa durante la segunda Guerra Mundial. Llama la atención de José Honório Rodrigues quien fue director del Instituto Brasileiro de Relações Internacionais entre 1964 y 1968.

Finalmente, referido a la adhesión ideológica en tres casos son autores vinculados al ecumenismo, siete al marxismo y dos eran teólogos de la liberación. La tabla siguiente lo detalla.

Tabla 3: Comparación de las trayectorias de militancia.

\begin{tabular}{|c|c|c|c|c|c|c|c|c|c|c|}
\hline \multirow{3}{*}{ País } & \multirow{3}{*}{ Autor } & \multicolumn{9}{|c|}{ Tópico dominante } \\
\hline & & \multirow{2}{*}{$\begin{array}{c}\begin{array}{c}\text { Estudios } \\
\text { de } \\
\text { posgrado }\end{array} \\
1\end{array}$} & \multicolumn{2}{|c|}{ Religión } & \multicolumn{3}{|c|}{ Política } & \multicolumn{3}{|c|}{ Ideología } \\
\hline & & & 2 & 3 & 4 & 5 & 6 & 7 & 8 & 9 \\
\hline Argentina & Néstor García Canclini & $\mathrm{X}$ & $\mathrm{X}$ & - & - & - & - & $\mathrm{X}$ & $\mathrm{X}$ & - \\
\hline Austria & Ivan Illich & $\mathrm{X}$ & $\mathrm{X}$ & $\mathrm{X}$ & - & - & - & $\mathrm{X}$ & - & - \\
\hline Bélgica & Conrado Detrez & - & - & - & $\mathrm{X}$ & $\mathrm{X}$ & $\mathrm{X}$ & - & $\mathrm{X}$ & - \\
\hline \multirow{11}{*}{ Brasil } & Moacyr Felix & $\mathrm{X}$ & - & - & $\mathrm{X}$ & $\mathrm{X}$ & $\mathrm{X}$ & - & $\mathrm{X}$ & - \\
\hline & José Honório Rodrigues & $\mathrm{X}$ & - & - & - & - & - & - & - & - \\
\hline & Waldo Cesar & $\mathrm{X}$ & $\mathrm{X}$ & - & $\mathrm{X}$ & - & $\mathrm{X}$ & $\mathrm{X}$ & $\mathrm{X}$ & - \\
\hline & Luiz Alberto Gómes De Sousa & $\mathrm{X}$ & $\mathrm{X}$ & - & $\mathrm{X}$ & - & - & $\mathrm{X}$ & - & - \\
\hline & Luiz Eduardo Wanderley & $\mathrm{X}$ & $\mathrm{X}$ & - & - & - & - & $\mathrm{X}$ & $\mathrm{X}$ & - \\
\hline & José Leite Lopes & $\mathrm{X}$ & - & - & $\mathrm{X}$ & $\mathrm{X}$ & - & - & $\mathrm{X}$ & - \\
\hline & Jovelino Pereira Ramos & $\mathrm{X}$ & - & $\mathrm{X}$ & $\mathrm{X}$ & $\mathrm{X}$ & - & $\mathrm{X}$ & - & - \\
\hline & Henrique De Lima Vaz & $\mathrm{X}$ & - & $\mathrm{X}$ & - & - & - & - & - & - \\
\hline & Frei Eliseu Lopes & - & - & $\mathrm{X}$ & - & - & - & $\mathrm{X}$ & - & - \\
\hline & Helder Câmara & - & - & $\mathrm{X}$ & $\mathrm{X}$ & - & - & $\mathrm{X}$ & - & $\mathrm{X}$ \\
\hline & Maria Helena Kühner & - & - & - & $\mathrm{X}$ & - & - & - & $\mathrm{X}$ & - \\
\hline EE.UU. & Richard Shaull & $\mathrm{X}$ & $\mathrm{X}$ & $\mathrm{X}$ & $\mathrm{X}$ & $\mathrm{X}$ & - & $\mathrm{X}$ & - & $\mathrm{X}$ \\
\hline \multirow{2}{*}{ Francia } & André Dumas & - & - & $\mathrm{X}$ & - & - & - & $\mathrm{X}$ & - & - \\
\hline & Paul Ricoeur & - & - & - & - & - & $\mathrm{X}$ & $\mathrm{X}$ & - & - \\
\hline Suiza & Pierre Furter & $\mathrm{X}$ & $\mathrm{X}$ & - & - & - & - & $X$ & - & - \\
\hline Uruguay & Hiber Conteris & $\mathrm{X}$ & $\mathrm{X}$ & - & $\mathrm{X}$ & $\mathrm{X}$ & $\mathrm{X}$ & $\mathrm{X}$ & - & - \\
\hline
\end{tabular}

Referencias: 1) posgrado, 2) cargos ecuménicos, 3) jerarquía religiosa (pastor, sacerdote, pastor), 4) perseguido, 5) exiliado, 6) preso, 7) ecuménico cristiano, 8) marxista y 9) teólogo liberacionista. 


\section{BIBLIOGRAFÍA}

ACADEMIA BRASILEIRA DE LETRAS (S/F) “José Honório Rodrigues. Biografía”. www.academia.org.br/abl/cgi/cgilua.exe/sys/start.htm?infoid=749\&sid=323 (consultado 7/5/2015).

AMAT Y LEÓN, Oscar. Impacto del ecumenismo en Perú, Lima: Pontificia Universidad Católica del Perú, 1996.

CARUSO, Francisco. “José Leite Lopes: In Memoriam” Mens Agitat Vol. IV, N․1, 2009, p. 123-126

CERVANTES-ORTIZ, Leopoldo "Hiber Conteris en sus ochenta años" Protestante Digital 9/02/2014.

http://protestantedigital.com/magacin/14240/Hiber_Conteris_en_sus_ochenta_antildeos (consultado 23/5/2015).

CESAR, Waldo "Entrevista: Sociólogo relembra a abertura dos evangélicos para a realidade social brasileira nos anos 60" Ultimato, marzo-abril 2007, $\mathrm{N}^{\circ} 305$.

http://www.ultimato.com.br/revista/artigos/305/sociologo-relembra-a-abertura-dosevangelicos-para-a-realidade-social-brasileira-nos-anos-60 (consultado 2/6/2015).

CONCATTI, Rolando. Testimonio cristiano y resistencia en las dictaduras argentinas. El movimiento ecuménico en Mendoza 1963-1983. Buenos Aires: Centro Nueva Tierra, 2009.

CUNHA, Magali do Nascimento "O passado nunca está morto. Um tributo a Waldo César e sua contribuição ao movimento ecumênico brasileiro Magali do Nascimento Cunha", Estudos de Religião, jul/dez 2007, Ano XXI, 33, pp.136-158.

CZAJKA, Rodrigo "O comando dos trabalhadores intelectuais e a formação das esquerdas culturais na década de 1960", Temáticas, 19 (37/38) Jan./dez. 2011, pp. 57-80.

DA MATA, Sérgio (2007) “A dialética do aggiornamento", Revista do Arquivo Público Mineiro, №2, jul-dic/2007, 144-157.

DEVÉS, Eduardo. "La circulación de las ideas, una conceptualización: el caso de la teología latinoamericana en Corea del Sur". Estudios Avanzados, 2016, N² 25, pp. 20-41.

DOMINGUES DE ALMEIDA, José “Conrad Detrez (1937 - 1985)”, Ulyssei@s, 2015, http://ulysseias.ilcml.com/pt/termos/detrez-conrad/ (consultado 25/10/2015).

EDITORES "Waldo César: vida e compromisso com a responsabilidade social da igreja", Novos Diálogos, 20/6/2011, http://www.novosdialogos.com/artigo.asp?id=596 (consultado, $22 / 9 / 2014)$.

HASSAN MONTERO, Yusef; HERRERO SOLANA, Víctor y GUERRERO - BOTE, Vicente "Usabilidad de los tag-clouds: Estudio mediante eyetracking" Scire. Representación y organización del conocimiento. 2010, Vol.16 N. 1, pp. 15-33.

INFOAMÉRICA (S/F) Néstor García-Canclini (1939-). Perfil biográfico y académico. www.infoamerica.org/teoria/garcia_canclini1.htm (consultado 1/3/2016).

KÜHNER, Maria Helena (2003) Pequeno memorial descritivo de sua formação e sua carreira, www.kuhner.com.br/mariahelena/ (consultado 25/10/2015). 
LAFUENTE, M. Avelina Cecilia “Introducción al pensamiento de Paul Ricoeur”, Thémata, 1998, N. 19 , pp. 219-223.

LEITE LOPES, J. S. “Fabrica e vila operária: considerações sobre uma forma de subordinação burguesa". In: Leite Lopes, S. et al. Mudança Social no Nordeste: a reprodução da subordinação. Rio de Janeiro: Paz e Terra, 1979.

MAC DOWELL, João A. "In Memoriam - Padre Henrique Cláudio de Lima Vaz SJ (1921 - 2002)". Jesuitas Brasil. 2002.

www.padrevaz.com.br/index.php?option=com_content\&view=article\&id=59:in-memoriamjoao-a-mac-dowell\&catid=64: biografia-e-fotos\&Itemid=79 (consultado 4/5/2016).

MINDÊLO, Olívia "Você sabe quem foi Padre Henrique?", Prefeitura do Recife www.recife.pe.gov.br/pr/seceducacao/especial/padreHenrique/quem.html (consultado 12/5/2016).

OLIVERA, Ademar, Forjando caminos de liberación. La Iglesia Metodista en tiempo de dictadura, Montevideo: Trilce. 2009.

PAREDES, Alejandro "Paralelismos y Diferencias en la Práctica Ecuménica: Un Análisis de los Vínculos entre América Latina y Asia en la Red de Publicaciones de Mauricio López (19661972)". Asian Journal of Latin American Studies, 2013, N. 26 (2), pp.103-129.

PIMENTEL, Layana Karine "Paz e Terra: o pensamento da esquerda cristã expresso nos debates da revista (1966 a 1969)" Revista Brasileira de História das Religiões, 2009, v. 1, n. 3.

http://www.dhi.uem.br/gtreligiao/rbhr/paz_e_terra_o_pensamento_da_esquerda_crista.pdf (consultado 12/7/2016).

RIGHTEOUS AMONG THE NATIONS DEPARTMENT, archivo M.31.2/6368, 1994 http://db.yadvashem.org/righteous/righteousName.html?language=en\&itemId=4042816 (consultado 21/9/2015).

S/A "Antônio Henrique", Prefeitura do Recife, www.recife.pe.gov.br/pr/seceducacao/especial/padreHenrique/peantonio.html (consultado 12/5/2016).

S/A “Dominicano vê a ação da igreja no mundo moderno" Correio da manhã, 21/07/1966, p.7.

S/A "José Leite Lopes", Universidade Federal de Campina Grande,

www.dec.ufcg.edu.br/biografias/JosLeLop.html (consultado 5/6/2016).

S/A “Poeta Moacyr Félix de Oliveira morre aos 79 anos no Rio", Folha de São Paulo, 26/10/2005.

SMITH, Christian (1991) The Emergence of Liberation Theology: Radical Religion and Social Movement Theory, Chicago: University of Chicago Press.

SOTO, Eriberto “Semblanza de Richard Shaull”, Teología y cultura, año 8, vol. 13 (nov. 2011); pp.65-66.

TOURIS, Claudia "Luiz Alberto Gómez de Souza: En la Iglesia brasileña hay cierta involución hacia lo eclesiástico que es diferente de lo eclesial: la comunidad", Criteri, № 2363, Septiembre 2010, http://www.revistacriterio.com.ar/iglesia/luiz-alberto-gomez-de-souza-\%E2\%80\%9Cen-la- 
iglesia-brasilena-hay-cierta-involucion-hacia-lo-eclesiastico-que-es-diferente-de-lo-eclesial-lacomunidad\%E2\%80\%9D/ (consultado 12/7/2016).

UHLY, Steven "Paulo César Fonteles de Lima - Poesia e Ditadura", 2006, Revista Eletrônica Literatura e Autoritarismo, 9 (1),

www.ufsm.br/literaturaeautoritarismo/revista/num09/art_01.php (consultado 2/9/2015) 